\title{
Municipal Solid Waste, Market Competition and the EU Policy
}

\author{
Carlo Reggiani $^{1}$ (iD) - Francesco Silvestri ${ }^{2}$
}

Accepted: 28 May 2017 / Published online: 8 June 2017

(C) The Author(s) 2017. This article is an open access publication

\begin{abstract}
Two of the main pillars of the EU solid waste policy are the Proximity Principle and the Self-Sufficiency Principle. According to those, waste should be disposed as close as possible to where it has been produced. A likely effect of such provision is to prevent competition from neighbouring areas and increase the market power of local disposers, with possible undesirable consequences for other firms in the vertical chain. We show through a simple spatial model that one additional effect of the Proximity Principle and of the SelfSufficiency Principle is to provide an incentive to collectors and waste producers to increase the amount of separated waste.
\end{abstract}

Keywords EU municipal waste policy $\cdot$ Self-Sufficiency Principle $\cdot$ Proximity Principle

JEL Classification Q53 - L13 · L44

\section{Introduction}

Municipal Solid Waste (MSW) is the waste generated by households. In the European Union (EU), local bodies at the municipality level are legally obliged to provide a collection system for MSW, in agreement with the regulations and guidelines set by EU directives, national laws, and regional plans. The EU directives posit that MSW should be handled and disposed

We would like to thank Prasenjit Banerjee and Giacomo Calzolari, the co-editor Sheila Olmstead and two anonymous referees for helpful comments. The usual disclaimer applies.

Carlo Reggiani

carlo.reggiani@manchester.ac.uk

Francesco Silvestri

slvfnc1@unife.it

1 School of Social Sciences - Economics, University of Manchester, Manchester M13 9PL, UK

2 Department of Economics and Management, University of Ferrara, Via Savonarola 9, 44121 Ferrara, Italy 
close to the location where it is generated: to achieve this objective, each EU member state designs territorial areas where to treat and dispose MSW generated by the local households. This policy is rooted in the two pillars of Self-Sufficiency Principle (SSP) and Proximity Principle (PP). These principles impose the establishment of an integrated network of municipal waste treatment facilities in order to ensure to each community a self-sufficient disposal and recovery capacity, such that waste is processed in the proximity of where it is generated. The resulting policy is aimed to reach two main goals: the first is to prevent the creation of pollution havens in regions with low environmental standards; the second is to enhance local communities' awareness of their environmental responsibilities. ${ }^{1}$ From an economic viewpoint, however, the policy has also an impact on the market structure of the waste sector. The current regulation, in fact, limits the choices of the local collectors, that cannot transport waste to disposal facilities in neighbouring areas. As a result, local disposers face a more limited number of competitors (if any) and, as a result, are likely to have more market power.

The MSW industry has a vertical structure, reflecting the three main phases of MSW processing. In the first phase households generate $\mathrm{MSW}^{2}$ The second phase is the collection of MSW: a municipality based collector picks up waste from the households. In this phase households do exert some effort to separate recyclable from undifferentiated waste. This is an important activity reflecting another principle on which the current EU regulation is based: the Extended Producer Responsibility (EPR). According to it, all subjects acting along the goods production and retail chains are responsible for the final diversion of those goods when transformed into waste. As a consequence, the EPR and its reuse-recover targets potentially reduce the amount of waste that reaches disposal facilities. In the third and last phase, the collector brings the waste to a disposer that processes MSW: undifferentiated waste is either processed in a landfill or by an incinerator. Separated waste, instead, is recycled directly by the collector. Mainly due to scale and density economies, the collection phase can be considered a legal monopoly; moreover, the public/merit good nature of MSW advocates for the implementation of a compulsory public service. ${ }^{3}$ The disposal segment instead could in principle operate in a more competitive regime. The provisions of the SSP and of the $\mathrm{PP}$, however, force collectors to find a disposing facility inside a defined territorial area and among a restricted number of disposers, allowing the latter to potentially exploit a scarcity rent. Whereas the interrelationship between waste regulation and competition at the disposal segment is a more general issue, the EU case is of particular interest because of the inconsistency between the treatment of MSW vis à vis industrial and hazardous waste. It is noteworthy, in fact, that the same principles (the SSP and the PP) do not apply to industrial waste: despite the higher environmental hazard, the latter can be sent to any licensed disposal facility in the world, effectively allowing more competition between disposers.

In this paper, we aim to highlight the impact of regulation and, in particular of the SSP and of the PP, on the market outcomes in the EU waste sector with a particular focus on the resulting incentives for households and collectors to engage in waste separation and recycling. To this end we set up a simple spatial model of MSW production, collection and

\footnotetext{
1 The EU waste regulation is a complex body, whose framework is set in the European Parliament and Council Directive 2008/98/EC (http://ec.europa.eu/environment/waste/framework/). Silvestri (2014) provides a more detailed discussion of the current regulation and its principles.

2 It is worth noticing that a good part of the recent literature on MSW and separate disposal has focused on the interaction between local councils and households in this first phase. As it will become clear, our model captures all the phases of MSW processing but our focus is mainly on the collection and disposal ones. Please refer to footnote 5 for further discussion.

3 An inappropriate or inefficient collection causes significant negative externalities to the local community; see, for example, D'Alisa et al. (2010).
} 


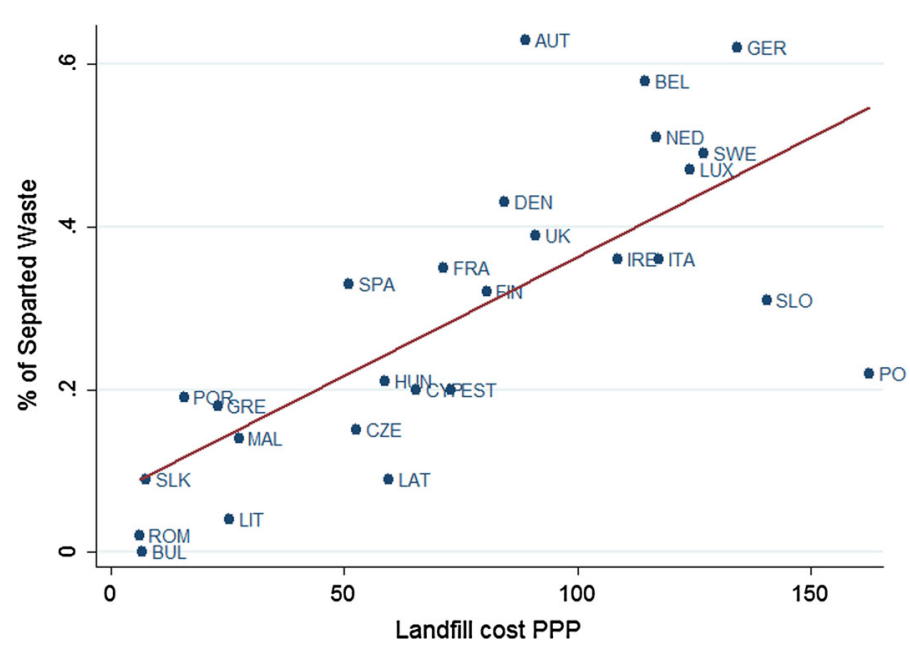

Fig. 1 Separated collection and the cost of landfill. Source: Eurostat (2010)

disposal. The model allows analyzing the major economic effects of the EU policy and, in particular, the costs and benefits of the SSP and PP provisions. The analysis provides relevant policy implications. First of all, we confirm that the SSP and PP principles of the EU waste regulation, limiting competition between disposers, increase the cost of MSW collection to the local community. Second, however, we also highlight a subtler consequence of such principles: the higher cost of disposing MSW leads households to exert more effort and, in turn, increase the amount of separated collection, decreasing instead the waste sent for disposal. The latter substitution effect may be a less intended implication of the current EU regulation and it constitutes, perhaps, a further rationale behind the imposition of the SSP and PP principles. Aggregate level evidence from the 27 EU countries suggests indeed a negative correlation between the percentage of unselected MSW processed by a disposer (landfill or incinerator) and the average disposing costs $(-0.65)$ and a positive correlation between the percentage of separated waste and the average disposing costs (0.73). The latter is illustrated in Fig. 1. As liberalization in other regulated sectors (e.g. gas, electricity) has led to a decrease in the final price to users, we may conjecture that a similar process in the MSW segment would increase the percentage of disposed waste and reduce households' effort for separated collection.

To the best of our knowledge, this is one of the first papers to model the MSW industry in all its segments and participating agents. Most of the existing literature on MSW and its management has focused on either: (i) the environmental implications of waste and policies to correct welfare distortions (Davies and Doble 2004; Jenkins et al. 2004; Caplan et al. 2006) or (ii) competition for the market (Demsetz 1968), according to which natural monopolies can be managed by private firms whose right to operate is entrusted by the government via competitive tendering (Williamson 1976; Laffont and Tirole 1994, Ch.7). We focus instead on competition in the MSW market (Porter and Linde 1995; Crocker and Masten 1996; Massarutto 2007). Furthermore, a stream of literature, initiated by the seminal work in Fullerton and Kinnaman (1995), highlights the role of price differences to environmental choices. The emphasis is on waste dumping, a prominent source of harsh environmental damage that has been further studied also by Choe and Fraser (1998, 1999), Shinkuma (2003), Almer and Goeschl (2010), D'Alisa et al. (2010), D'Amato and Zoli (2012), inter alia. Unlike most of 
this literature, we do model the vertical structure of the MSW sector. ${ }^{4}$ Moreover, our work complements the recalled literature by emphasizing a different channel through which price differences matter, i.e. the incentives for separated waste collection, and underscoring that such price differences arise in the EU due to the very peculiar regulatory structure of MSW. Furthermore, the model in this paper can be adapted to address a number of other relevant and related issues. For example, Silvestri (2014) focuses on related issues like: (a) other possible policy instruments to achieve the objectives of the current regulation but limiting the distortions; (b) the analysis of the implications of the sector's structure for the entrance of new disposers. Finally, whereas our paper addresses regulation in the European MSW sector, the implications of our analysis may go beyond and find a wider application in contexts where relaxing waste regulation has a likely "pro-competitive" effect on segments of the MSW chain.

The rest of the paper is structured as follows. In Sect. 2, we introduce a spatial model of the MSW sector, capturing many of the characterizing features of the EU case. The model is solved in Sect. 3 and the "status quo", in which the SSP and PP are enforced, is compared with a scenario in which the regulation is relaxed and the disposal sector is exposed to more competition. In Sect. 4, we focus on the surplus and welfare implications of our analysis. Section 5 provides an illustration based on the special case of symmetric areas. Finally, Sect. 6 briefly discusses the main results and concludes. All proofs are in the Appendix.

\section{A Spatial Model of MSW}

Consider two bordering geographical areas $(i=A, B)$; consistently with the previous discussion, in each area there are Households $(\mathrm{H})$, a monopolistic Collector $(\mathrm{C})$ and a disposing facility (D).

Households The households living in the local community of the area produce an amount of MSW $Q_{i}$ : for analytical convenience, we shall normalize $Q_{i}$ to $1 .^{5}$ According to the current legislation, all the MSW must be removed. A fraction $q_{i} \leq 1$ is unseparated waste. As a consequence of the EPR principle, the removal of separated MSW is free for households: this provides an incentive to put costly effort $e_{i}$ in separating waste. The previous discussion can be summarized by the following utility function of a given household:

$$
U_{i}^{H}=\bar{u}_{i}-p_{i} q_{i}-e_{i},
$$

where $p_{i}$ is the unit price paid by the household for the collection of unseparated MSW. The quantity of separated collection $d_{i}$ depends on the effort but also on the available selection capacity $k_{i}$, provided by the local collector. We shall assume that waste separation takes place according to the following Cobb-Douglas technology:

$$
d_{i}=e_{i}^{\frac{1}{2}} k_{i}^{\frac{1}{2}}, \quad d_{i} \leq 1
$$

\footnotetext{
4 Another stream of literature has carefully modelled the MSW sector to analyze the optimal design of solid waste management programs: competition and market structure, however, are not usually addressed. See Di Corato and Montinari (2014) for a recent contribution.

5 As our focus is the vertical structure of the MSW sector, our setup assumes there is a representative household in each area. Part of the extant literature, instead, focuses on the interaction between households and the area's authorities, with the latter imposing to the households the price of collection (Yang and Innes 2007; Reichenbach 2008; Hong et al. 1993) or the selection method (Czajkowski et al. 2016).
} 


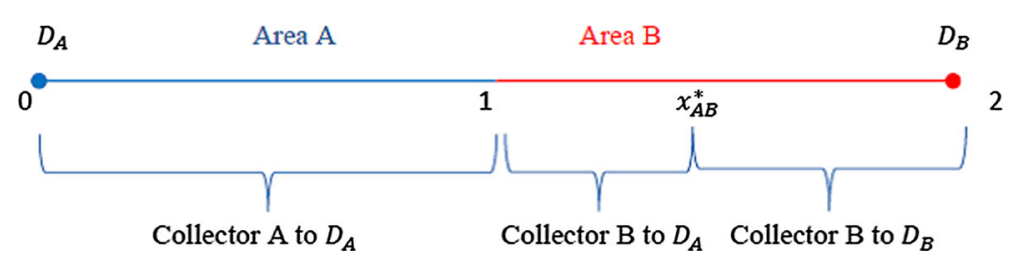

Fig. 2 An example of deregulated spatial waste market

In other words, separated waste is the output originating from three inputs: the raw material, $Q_{i}$, that we normalised to 1 , the household's effort, $e_{i}$, and the installed capacity for separated collection, $k_{i}$.

Collectors The monopolist collectors operate exclusively in their area; however, where waste is disposed depends on the regulatory regime. Under the current EU regime, unseparated waste must be disposed in the local area; on the contrary, if the provisions of the SSP and PP are relaxed, collectors have no obligation in disposing the picked unseparated waste in the local or the external landfill. The collector is chosen as a franchised or natural monopolist to operate in the collection segment. The collector also provides the facilities for households to separate waste: in particular, they install a selection capacity $k_{i}$ and investment in capacity has a quadratic cost. ${ }^{6}$ The (exogenous) unit price $p_{i}$ for unseparated waste is set by the regulator; ${ }^{7}$ similarly, the unit price for separated waste collection $\widetilde{p}_{i}$ is also exogenously set according to the EPR system.

We capture the geographical aspects, a defining characteristic of this sector, through a simple model à la Hotelling (1929). Area $A$ and $B$ are represented as unit length lines, so that the overall market has a total length of 2 (see Fig. 2). ${ }^{8}$ Households are uniformly distributed along the line in both areas, with $x_{i} \in[0,2]$ denoting the location of a specific household. Disposal facilities are located at the extremes of each line, i.e. disposer $A$ is located at $x=0$ and disposer $B$ at $x=2$. Collectors face linear transportation costs in picking-up unseparated waste and bringing it to the location of the facilities. ${ }^{9}$ We denote the total transport costs of gathering the waste and bringing it to the disposal facility as $T_{i}$.

Taking for reference the collector operating in area $A$, collector $A$ 's profit can be written as:

$$
\pi_{A}^{C}= \begin{cases}\left(p_{A}-a_{A}\right) q_{A} x_{A B}^{*}+\left(p_{A}-a_{B}\right) q_{A}\left(1-x_{A B}^{*}\right)+\tilde{p}_{A} d_{A}-k_{A}^{2}-T_{A}\left(x_{A B}^{*}\right) & \text { if } x_{A B}^{*}<1 \\ \left(p_{A}-a_{A}\right) q_{A}+\widetilde{p}_{A} d_{A}-k_{A}^{2}-T_{A}\left(x_{A B}^{*}\right) & \text { if } x_{A B}^{*}>1\end{cases}
$$

where $a_{i}$ are the costs of disposing the waste in area $i$ and $x_{A B}^{*}$ is the household that makes the collector is indifferent between bringing its waste to the local or to the external facility. In the context of our model, then $x_{A B}^{*}<1$ is the fraction of unseparated waste taken to the local disposer in area $A$, with the rest being brought for disposal to the external facility in area $B$; if, instead, $x_{A B}^{*}>1$, collector $A$ brings all unseparated waste to the local disposer. Clearly,

\footnotetext{
6 Quadratic costs are assumed for analytical convenience but any convex function would not affect our results.

7 The price $p_{i}$ may be set, for example, by a higher order social planner (regional or national authority) or fixed in the auction through which the franchised monopolist collector is appointed.

8 This normalization simplifies the exposition and it is without loss of generality: the length of each area could be any $L>0$ without affecting any of our conclusions.

9 For simplicity, we do not consider any intermediate recollection, such as transitional places where to accumulate and treat the unselected waste that come from households.
} 
under the current EU regulation, $x_{A B}^{*}=1$ is enforced and it is not a variable of choice of the collector. As it will become clear, if regulation is lifted, $x_{A B}^{*}$ will depend on the disposal prices at the two facilities and on the transport costs. The total transport costs depend on $x_{i}$ and, for example, it can be explicitly expressed as:

$$
T_{A}\left(x_{A B}^{*}\right)= \begin{cases}t q_{A}\left[\int_{0}^{x_{A B}^{*}} u d u+\int_{x_{A B}^{*}}^{2}(2-u) d u\right] & \text { if } x_{A B}^{*}<1 \\ t q_{A} \int_{0}^{1} u d u & \text { if } \quad x_{A B}^{*}>1\end{cases}
$$

Similar expressions hold for both the profits and transport costs of collector $B .^{10}$

Disposers. The disposing facility $D_{i}$ receives the unseparated MSW and charges $a_{i}$ for each unit to be disposed. Notice that $a_{i}$ is the same, no matter what is the origin of the waste. If the SSP and PP are relaxed, in fact, the facility may receive waste from both the local and the outside collectors: the disposers are not allowed to discriminate waste according to its origin. The profits of disposer $A$ are, then:

$$
\pi_{A}^{D}\left(a_{A}, a_{B}\right)=\left\{\begin{array}{lll}
a_{A}\left[q_{A}+q_{B}\left(x_{A B}^{*}-1\right)\right] & \text { if } & a_{A}<a_{B} \\
a_{A} q_{A} & \text { if } & a_{A}=a_{B} \\
a_{A} q_{A} x_{A B}^{*} & \text { if } & a_{A}>a_{B}
\end{array}\right.
$$

and the profits of disposer $B$ can be written in a similar way.

The timing of the game is as follows:

1. Disposers decide the amount charged, $a_{i}$, for the disposal of unseparated waste;

2. Collectors choose the selection capacity $k_{i}$ for separated collection and are obliged to collect any unseparated waste;

3. Households exert effort $e_{i}$ for separated collection and produce unseparated waste $q_{i}$.

\section{Analysis}

\subsection{The Choice of Effort}

Proceeding by backward induction, we start by solving for the households choices on the effort to be put into separated collection and the amount of unseparated waste. These choices hold for any level of the installed selection capacity by collectors and prices chosen by the disposers. Given (2.2) and the fact that $q_{i}=1-d_{i}$, the LC $i$ maximises utility (2.1). The optimal effort choice of a household in area $i$ is obtained for:

$$
e_{i}^{*}=\frac{k_{i} p_{i}^{2}}{4},
$$

which, in turn, implies that the unseparated collection is:

$$
q_{i}^{*}=1-\frac{k_{i} p_{i}}{2} .
$$

10 Transport costs are specified as an "on-demand" collection system, in line with the received literature on spatial models of product delivery (Lederer and Hurter 1986; Vogel 2011, inter alia). A "milk-run" circulating collection system may better approximate reality and can be assumed without affecting the model's conclusions as long as the (transport) costs of collection increase convexly in the total distance covered. 
According to (3.1) and rather intuitively, the effort exerted by households in separated collection is positively related to the selection capacity installed by the collector, $k_{i}$ and to the price of unseparated collection, $p_{i}$. A high collection price encourages the substitution of unseparated waste with separated one, as confirmed by (3.2). As households in different areas never interact, their choices are not directly affected by the regulation regime, so these results apply to both the regimes that we analyse.

\subsection{Collectors' Choices}

Whereas the EU regulation constitutes the current status quo, it arises a special case in our model: hence, we shall first analyze the case of no regulation. If no regulation is imposed, from (2.3), it is immediate to find that:

$$
x_{A B}^{*}=1+\frac{a_{B}-a_{A}}{2 t},
$$

i.e. whether it is collector $A$ or collector $B$ to bring unseparated waste to the disposer in a different area depends on the price differential, $a_{B}-a_{A}$. If the latter is non-negative, then $x_{A B}^{*} \geq 1$. We can then start by assuming, without loss of generality, that $x_{A B}^{*} \geq 1$. Given (3.1) and (3.2), collectors maximize profits $\pi_{A}^{C}$ and $\pi_{B}^{C}$ with respect to $k_{A}$ and $k_{B}$ respectively. The first order conditions are:

$$
\begin{aligned}
& \frac{\partial \pi_{A}^{C}}{\partial k_{A}}=\frac{-8 k_{A} p_{A} \sqrt{k_{A}}+p_{A}^{2} \sqrt{k_{A}}\left[2\left(a_{A}-p_{A}+\tilde{p}_{A}\right)+t\right]}{4 p_{A} \sqrt{k_{A}}}=0 \\
& \frac{\partial \pi_{B}^{C}}{\partial k_{B}}=\frac{-16 t k_{B} p_{B} \sqrt{k_{B}}-p_{B}^{2} \sqrt{k_{B}}\left[\left(a_{B}-a_{A}\right)^{2}-4 t\left(a_{B}-p_{B}+\widetilde{p}_{B}\right)-2 t^{2}\right]}{8 t p_{B} \sqrt{k_{B}}}=0
\end{aligned}
$$

Solving these simultaneously allows finding the optimal selection capacity to be installed for separated collection: ${ }^{11}$

$$
\begin{aligned}
& k_{A}^{*}=\frac{p_{A}}{8}\left[2\left(a_{A}-p_{A}+\tilde{p}_{A}\right)+t\right], \\
& k_{B}^{*}=\frac{p_{B}}{8}\left[2\left(a_{B}-p_{B}+\tilde{p}_{B}\right)+t\right]-\frac{p_{B}}{16 t}\left(a_{B}-a_{A}\right)^{2},
\end{aligned}
$$

and, consequently, the resulting unseparated collection:

$$
\begin{aligned}
& q_{A}^{*}=1-\frac{p_{A}^{2}}{16}\left[2\left(a_{A}-p_{A}+\tilde{p}_{A}\right)+t\right], \\
& q_{B}^{*}=1-\frac{p_{B}^{2}}{32}\left[4\left(a_{B}-p_{B}+\tilde{p}_{B}\right)+2 t-\frac{\left(a_{B}-a_{A}\right)^{2}}{t}\right] .
\end{aligned}
$$

Under the assumption $x_{A B}^{*} \geq 1$, collector $A$ brings all of the waste generated in area $A$ to the local disposer. Hence, his selection capacity choice is not directly affected by the price of disposing in area $B$. If the latter inequality is strictly satisfied, this is not the case for collector $B$. In absence of regulation and as the price of disposing in area $A$ is lower than in $B\left(a_{A}<a_{B}\right)$, then the waste of households located between 1 and $x_{A B}^{*}$ is brought for disposal to area $A$. The rest of the waste, gathered between $x_{A B}^{*}$ and 2, is disposed in area $B$. Clearly, then, the disposal price differential $a_{B}-a_{A}$ affects the choices of collector $B$, as it can be seen in (3.5) and (3.7). In particular, the last term in (3.5) shows that the higher the

11 It is also easy to verify that the second order conditions for a maximum hold. 
discrepancy of disposal prices in the two areas, the lower is the incentive for collector $B$ to install selection capacity.

If the EU regulation is imposed and $x_{A B}^{*}=1$, the optimal selection capacity and unseparated collection are, respectively:

$$
\begin{aligned}
& k_{i}^{E U}=\frac{p_{i}}{8}\left[2\left(a_{i}-p_{i}+\tilde{p}_{i}\right)+t\right], \\
& q_{i}^{E U}=1-\frac{p_{i}^{2}}{16}\left[2\left(a_{i}-p_{i}+\tilde{p}_{i}\right)+t\right] .
\end{aligned}
$$

Comparing (3.4) and (3.8) it is clear that, for a given price of disposal $a_{A}$, the choice of collector $A$ is not affected by the regulatory regime.

\subsection{Disposers' Choices}

In absence of EU regulation, the disposers maximize their profits given the choices of the households, (3.1), and of the collectors, (3.4)-(3.7). As we focus on the case $x_{A B}^{*} \geq 1$, the profits functions of the disposers are, respectively:

$$
\begin{aligned}
& \pi_{A}^{D}\left(a_{A}, a_{B}\right)=a_{A}\left[q_{A}^{*}+q_{B}^{*}\left(x_{A B}^{*}-1\right)\right] \\
& \pi_{B}^{D}\left(a_{A}, a_{B}\right)=a_{B} q_{B}^{*}\left(2-x_{A B}^{*}\right)
\end{aligned}
$$

According to the previous assumption on $x_{A B}^{*}$, disposer $A$ receives the unseparated waste from both areas, whereas disposer $B$ focuses on the remaining local waste.

In case EU regulation is holding and $x_{A B}^{*}=1$ is enforced, no waste can be transferred from one area to the other; as a consequence, the profit functions are:

$$
\pi_{i}^{D}=a_{i} q_{i}^{E U}
$$

First, we can fully characterize the equilibrium if the SSP and PP principles hold. The following lemma highlights the disposers' price choices.

Lemma 1 The disposers' price choices in presence of the EU regulation are:

$$
a_{i}^{E U}=\frac{1}{4}\left[\frac{16}{p_{i}^{2}}+2 p_{i}-2 \widetilde{p}_{i}-t\right] .
$$

Lemma 1 shows that under regulation the disposers' price choices in each area are completely independent. In other words, EU regulation isolates local disposers from the competition of disposers from other areas. The equilibrium price of disposal is affected by the parameters of the model in a complex way. First, $a_{i}^{E U}$ depends negatively on the price of separated collection, $\widetilde{p}_{i}$, and on the transport cost, $t$. The price of disposal also depends nonmonotonically on the price of unseparated collection: $a_{i}^{E U}$ increases if the collection price is sufficiently high. These effects depend on the way the parameters influence the collector's choice for selection capacity and how this, in turn, impacts on the demand for unseparated collection, $q_{i}^{E U}$ and, consequently, disposal.

We can then turn our attention to the effects of abandoning regulation and the SSP and PP principles. In this case we cannot fully characterize the equilibrium and provide the equilibrium expressions for the prices of disposal. However, the following results can be stated.

Proposition 2 (a) If in presence of the EU regulation the equilibrium prices were identical, $a_{A}^{E U}=a_{B}^{E U}$, abandoning regulation leads to a decrease in the disposal prices $a_{i}^{*}$ in both areas, $i=A, B$; 
(b) if $a_{A}^{E U}<a_{B}^{E U}$, a sufficient condition for the disposal price of A to decrease if regulation is abandoned is: $a_{A}^{E U}>a_{B}^{E U} / 2$. The disposal price of $B$ decreases.

(c) If abandoning EU regulation leads to lower disposal prices, then the incentives to build capacity for separated collection, $k_{i}^{*}$, to exert effort $e_{i}^{*}$ and the overall amount of separated collection $d_{i}^{*}$ are reduced.

Proposition 2 states the main results of the paper. In particular, part (a) and (b) identify the effects of relaxing the prescriptions of the current EU regulation on waste collection and disposal.

Part (a) establishes that if two areas, regions or countries have very similar prices of disposal in the current status-quo, opening the market for disposal and giving the possibility to collectors to transport waste to other neighbouring areas would lead to a reduction in the prices of disposal. The intuition is very simple: a small unilateral decrease in the price of disposal from $a_{i}^{E U}$ would increase profits as it allows to extend the market size. In other words, the collector from the neighbouring area would consider bringing a small share of the waste, produced by households located near the border, to the disposal facility that reduced the price. As both disposers face this pressure to reduce prices, the resulting unregulated equilibrium features lower prices in both areas. Allowing disposal in other areas has a "pro-competitive effect" that decreases the cost of the disposal of unseparated waste in both areas.

Part (b) extends the result to the case in which areas are heterogeneous, which is reflected in a different price of disposal, $a_{i}^{E U}$, in the regulated status quo. In particular, the result establishes that the "pro-competitive effect" of abandoning regulation is very likely to take place also in this case. A sufficient, but not necessary, condition for the price of disposal to decrease is that areas are not too heterogeneous and, more precisely, that the status-quo disposal prices are not too different. If, as we assumed, area $A$ is characterized by a lower disposal price, the pro-competitive effect of abandoning EU regulation prevails if the price is more than half of the price in area $B$, i.e. $a_{A}^{E U}>a_{B}^{E U} / 2$. Moreover, the higher price disposer, $D_{B}$, is surely going to decrease its price in response to deregulation. Hence, unless areas are particularly heterogeneous, part (b) shows that it is very likely that the prices of disposing unseparated waste decrease when waste can travel across areas.

The intuition for this second result is more intricate. In particular, as derived in the Appendix in (A.3), the effect of unilaterally decreasing the disposal price in area $A$ following a lift of the EU regulation can now be written as:

$$
\begin{aligned}
\left.\frac{\partial \pi_{A}^{D}}{\partial a_{A}}\right|_{a_{A}=a_{A}^{E U}}= & \underbrace{F O C_{A}^{E U}\left(a_{A}^{E U}\right)}_{=0}+\underbrace{a_{A}^{E U} q_{B}^{E U} \frac{\partial x_{A B}^{*}}{\partial a_{A}}}_{\text {Pro-competitive effect }}+\underbrace{a_{A}^{E U} \frac{\partial q_{B}}{\partial a_{A}}\left(x_{A B}^{*}-1\right)}_{\text {Indirect effect on } q_{B}} \\
& +\underbrace{q_{B}^{E U}\left(x_{A B}^{*}-1\right)}_{\text {Infra-marginal gain }} .
\end{aligned}
$$

In (3.14) there are three new terms compared to the case EU regulation still holds. The usual "pro-competitive effect", identified in part (a), is now captured by the second term and it pushes down the disposal price of $A$. However, there are now two extra terms. The third term captures the negative relation between the unseparated waste in $B, q_{B}$, and the price of disposal $a_{A}$ : as a share of waste is "exported" from $B$ to $A$, a higher disposal price of $A$ increases the average disposal cost in area $B$ and that acts to decrease the household production of unseparated waste. This effect, that we shall call "indirect effect" on $q_{B}$, clearly goes in the same direction of the "pro-competitive effect". The fourth term of (3.14), instead, captures the "infra-marginal gain" in profits due to abandoning regulation. This is related 
to the new market share obtained, as with no regulation $x_{A B}^{*}>1$, and clearly encourages disposer $A$ to increase its price. The complex balance of these three effects determines whether the price of disposal will decrease or not in area $A$ : the condition provided on the prices of disposal establishes when the "pro-competitive" and "indirect" effects are surely dominating the "infra-marginal gain". Matters are much simpler when looking at disposer $B$ : in that case all the effects univocally point in the direction of a price decrease.

Finally, part (c) establishes the effects of a possible decrease in the price of disposing unseparated waste. Given the comparative statics obtained in the previous stages of the game it is simple to see that the "pro-competitive effect" identified at the disposal layer of the waste market has an important consequence: higher competition in disposal can reduce and hinder the amount of resources dedicated to separated waste (selection capacity and household effort), leading to an overall decrease of the separation achieved by the local communities.

\section{Surplus and Welfare Implications}

The model presented in the previous sections is rather stylized as it has two main goals: first, to capture the vertical structure of the waste sector and, second, to focus on the impact of the EU waste policy on the incentives to engage in separated collection. To achieve those goals in the clearest possible way, we took a partial equilibrium perspective and, as such, a number of other relevant issues had to be neglected: a prominent example is illegal dumping, an activity that can cause very harsh environmental damage. Notwithstanding the simplifying assumptions and keeping in mind the limitations of our approach, it is interesting to briefly analyze the surplus and welfare implications of EU regulation. In particular, we try to decompose all effects of regulation and evaluate whether the benefits of increased separated disposal and recycling compensate for the losses associated to a more concentrated market.

Before setting off, it is worth recalling that in our model the total surplus in any given area is the sum of the households' indirect utility, the profits of the collector and of the disposer. The total surplus generated by the waste sector, however, does not coincide with welfare in this setting. The revenue obtained from recycling separated waste, $\widetilde{p}_{i} d_{i}$ in our notation, is extracted from firms or other agents that are exogenous to our (partial equilibrium) model of the waste sector. As such, that revenue constitutes a transfer that increases the surplus of the sector but does not impact on social welfare as a whole.

The surplus of area $i$ under EU regulation, after some simplification, can be written as:

$$
T S_{i}^{E U}=\bar{u}_{i}-e_{i}^{E U}-k_{i}^{E U 2}-\frac{t}{2} q_{i}^{E U}+\tilde{p}_{i} d_{i}^{E U}-C\left(q_{i}^{E U}\right),
$$

where $C$ (.) is the (non-decreasing) cost of the externality generated by disposing unseparated waste. The corresponding welfare is $W_{i}^{E U}=T S_{i}^{E U}-\widetilde{p}_{i} d_{i}^{E U}$ as by previous discussion.

If regulation is instead relaxed and, for example, $x_{A B}^{*} \leq 1$ then the surplus generated in area $A$ and area $B$ are, respectively:

$$
\begin{aligned}
& T S_{A}^{*}=\bar{u}_{A}-e_{A}^{*}-k_{A}^{* 2}-t q_{A}^{*}\left(x_{A B}^{* 2}-2 x_{A B}^{*}+\frac{3}{2}\right)+\tilde{p}_{A} d_{A}^{*}-C\left(x_{A B}^{*} q_{A}^{*}\right)-a_{B}^{*} q_{A}^{*}\left(1-x_{A B}^{*}\right), \\
& T S_{B}^{*}=\bar{u}_{B}-e_{B}^{*}-k_{B}^{* 2}-\frac{t}{2} q_{B}^{*}+\tilde{p}_{B} d_{B}^{*}-C\left(q_{A}^{*}\left(1-x_{A B}^{*}\right)+q_{B}^{*}\right)+a_{B}^{*} q_{A}^{*}\left(1-x_{A B}^{*}\right) .
\end{aligned}
$$

where the last term in both (4.2) and (4.3) is the transfer from collector $A$ to "export" waste to disposer $B$. 
The overall impact of EU regulation is obtained bringing together (4.1), (4.2) and (4.3):

$$
\begin{aligned}
T S^{E U}-T S^{*} & \left(T S_{A}^{E U}-T S_{A}^{*}\right)+\left(T S_{B}^{E U}-T S_{B}^{*}\right) \\
= & -\underbrace{\left[\left(e_{A}^{E U}+e_{B}^{E U}\right)-\left(e_{A}^{*}+e_{B}^{*}\right)\right]}_{(\mathrm{a})}-[\underbrace{\left[\left(k_{A}^{E U 2}+k_{B}^{E U 2}\right)-\left(k_{A}^{* 2}+k_{B}^{* 2}\right)\right]}_{(\mathrm{b})} \\
& -\underbrace{\left\{\frac{t}{2}\left(q_{A}^{E U}+q_{B}^{E U}\right)-\left[t q_{A}^{*}\left(x_{A B}^{* 2}-2 x_{A B}^{*}+\frac{3}{2}\right)+\frac{t}{2} q_{B}^{*}\right]\right\}}_{(\mathrm{d})} \\
+ & \underbrace{\left[\tilde{p}_{A}\left(d_{A}^{E U}-d_{A}^{*}\right)+\tilde{p}_{B}\left(d_{B}^{E U}-d_{B}^{*}\right)\right]} \\
- & \underbrace{\left\{\left[C\left(q_{A}^{E U}\right)+C\left(q_{B}^{E U}\right)\right]-\left[C\left(q_{A}^{*}\left(x_{A B}^{*}\right)+C\left(q_{A}^{*}\left(1-x_{A B}^{*}\right)+q_{B}^{*}\right)\right]\right\}\right.} .
\end{aligned}
$$

(e)

We can then identify the forces that drive the effects of EU regulation on the total joint surplus of areas $A$ and $B$. Suppose that, in equilibrium, the market fundamentals are such that the prices of disposal are higher in both areas if EU regulation is in place, i.e. $a_{i}^{E U}>a_{i}^{*}$ (Proposition 2b). From Proposition 2, part (c), the latter also implies $e_{i}^{E U}>e_{i}^{*}, k_{i}^{E U}>k_{i}^{*}$, $d_{i}^{E U}>d_{i}^{*}$ and $q_{i}^{E U}<q_{i}^{*}$. Hence, from (4.4), EU regulation has the following effects ${ }^{12}$ on the joint surplus:

(a) to increase the cost of effort that both LCs put in separating waste (-);

(b) to increase both collectors' investment in facilities for separated collection (-);

(c) if $x_{A B}^{*}<1$, to decrease the overall costs related to the transport of the unseparated waste $(+)$.

(d) to increase the revenue from the recycling of separated waste in both areas (+);

(e) to decrease the impact on the environmental externality of disposing unseparated waste $(+)$.

The overall welfare impact is obtained from (4.4) by subtracting the term (d): the revenue from recycling is a pure transfer and must be disregarded.

Clearly, it is extremely hard to reach general conclusions on the surplus and welfare effects and desirability of the current EU waste policy. Any answer depends on the balance between the positive and negative effects that were highlighted in the previous analysis. However, we can try to get some insight, for example by asking what are sufficient conditions under which EU regulation is welfare and/or surplus enhancing. To answer the question we use a revealed preference argument. Separated collection nowadays is heavily promoted and encouraged by the authorities at all levels around the globe; from this fact we can infer that for the local decision maker the benefits of separation (reducing the externality and possibly the costs and hazards of transporting waste) more than compensate for the costs of it (investment and effort required to engage in separation). ${ }^{13}$ In other words, if separation takes place welfare

12 The sign after each effect indicates the impact on the surplus differential.

13 We note that the argument requires that decision makers in any area take welfare maximizing decisions for the collectivity and they are immune from bounded rationality, political economy pressures or other sources of biased choice. 
and, a fortiori, surplus are positive in all areas and under all regimes. If we take the argument one step further and assume that the difference in benefits and costs of separation increases more than proportionally as the amount of unseparated waste and externalities decreases, then the welfare and surplus differentials display increasing differences and any policy that reduces externalities is also going to be welfare and surplus enhancing. In other words, a local decision maker whose interests are aligned with the ones of the collectivity and a benefit-cost differential displaying increasing differences are sufficient, although not necessary, conditions for the desirability of regulation.

In Sect. 5.1 we provide a more precise evaluation of the welfare and surplus effects of the EU regulation in the interesting special case of symmetric areas.

\section{A Special Case: Symmetric Areas}

We now focus on the special case of symmetric areas. This case may be of particular interest when considering regions with similar characteristics and it has the further advantage that equilibria in both scenarios can be characterized, which may be appealing for possible empirical applications of the model. ${ }^{14}$

If areas $A$ and $B$ are symmetric we have: $p_{A}=p_{B}=p, \widetilde{p}_{A}=\tilde{p}_{B}=\tilde{p}$ and $x_{A B}^{*}=1$. The disposal equilibrium price under EU regulation is still:

$$
a^{E U}=\frac{1}{4}\left[\frac{16}{p^{2}}+2 p-2 \tilde{p}-t\right] .
$$

If regulation is lifted, instead, the disposers choose the equilibrium price:

$a^{*}=\frac{1}{4}\left(\frac{16}{p^{2}}+2 p-2 \widetilde{p}+7 t\right)-\frac{\sqrt{4\left(p^{3}-p^{2} \tilde{p}+8\right)^{2}-4 p^{2} t\left(p^{3}-p^{2} \tilde{p}+8\right)+65 p^{4} t^{2}}}{4 p^{2}}$.

As a corollary of Proposition 2, the next result follows:

Corollary 3 If areas A and B are symmetric, abandoning EU regulation leads to a decrease in the prices of disposal: $a^{*}<a^{E U}$. The latter implies a reduction in the incentives to build capacity for separated collection, $k^{*}$, to exert effort $e^{*}$ and, ultimately, the overall amount of separated collection, $d^{*}$.

The corollary reinforces the message of Proposition 2. Relaxing the current EU regulation on waste management would make the disposer's segment of the market more competitive, with a decrease in the prices paid for disposal. However, the substitution between unseparated and separated collection lowers the collectors' incentives to invest in selection capacity. This, in turn, has a negative effect on the effort exerted by households, resulting in a lower level of unseparated collection.

\subsection{Surplus and Welfare}

Given the trade-off identified between disposal prices and separated collection, it is of interest to evaluate the surplus and welfare effects also if areas are symmetric. In this case the total surplus differential (4.4) simplifies to:

$$
T S^{E U}-T S^{*}=-2\left(e^{E U}-e^{*}\right)-2\left(k^{E U 2}-k^{* 2}\right)-t\left(q^{E U}-q^{*}\right)
$$

14 Silvestri and Ghinoi (2015) applies an adapted version of this model to waste management in the region of Lombardy in Italy. 


$$
+2 \tilde{p}\left(d^{E U}-d^{*}\right)-2\left[C\left(q^{E U}\right)+C\left(q^{*}\right)\right] .
$$

As the model has a closed form solution, in this case the effects highlighted in Sect. 4 can be more precisely identified. In particular, we can write the overall surplus and welfare differentials as, respectively:

$$
\begin{aligned}
& T S^{E U}-T S^{*}=\underbrace{\frac{p}{8}\left(a^{*}-a^{E U}\right)\left(a^{E U}+a^{*}-p\right)}_{\mathrm{A}}-\underbrace{2\left[C\left(q^{E U}\right)-C\left(q^{*}\right)\right]}_{\mathrm{C}}, \\
& W^{E U}-W^{*}=\underbrace{\frac{p}{8}\left(a^{*}-a^{E U}\right)\left(a^{E U}+a^{*}+2 \tilde{p}-p\right)}_{\mathrm{B}}-\underbrace{2\left[C\left(q^{E U}\right)-C\left(q^{*}\right)\right]}_{\mathrm{C}} .
\end{aligned}
$$

To gain insight about (5.1) and (5.2), it is worth recalling that by Corollary 3 the disposal prices are such that $a^{E U}>a^{*}$ and hence both terms $\mathrm{A}$ and $\mathrm{B}$ are negative. The equations also confirm that if EU regulation enhances welfare, it also leads to a higher total surplus: this is because in absolute value term B is larger than $\mathrm{A}$. Term $\mathrm{C}$ in both expressions positively contributes to the surplus and welfare differentials, as regulation allows reducing externalities. If the areas are symmetric, then, the surplus and welfare desirability of regulation boils down to the relative intensity of terms $\mathrm{A}$ and $\mathrm{B}$ as compared to $\mathrm{C}$.

The exact balance between those terms and, hence, the surplus and welfare consequences of regulation depend on the functional form used to model the environmental cost imposed to society by the disposal of unseparated waste. For example, the cost of the externality imposed maybe linear, e.g. $C\left(q_{i}\right)=c q_{i}$, or quadratic, e.g. $C\left(q_{i}\right)=c q_{i}^{2}$, in the amount of unseparated waste. We consider these two possible functional form and state the following result:

Proposition 4 If areas $A$ and $B$ are symmetric:

(a) either (i) EU regulation always leads to a higher surplus or (ii) there is a threshold value $\widehat{c}_{T S}>0$ such that EU regulation leads to a higher surplus iff $c \geq \widehat{c}_{T S}$;

(b) either (i) EU regulation always leads to a higher welfare or (ii) there is a threshold value $\widehat{c}_{W}>0$ such that EU regulation leads to a higher welfare iff $c \geq \widehat{c}_{W}$.

The threshold values are:

$$
\begin{aligned}
\widehat{c}_{T S} & =\frac{a^{*}+a^{E U}-p}{2}, \\
\widehat{c}_{W} & =\frac{a^{*}+a^{E U}-p+2 \widetilde{p}}{2},
\end{aligned}
$$

if the environmental cost is linear or:

$$
\begin{aligned}
\widehat{c}_{T S} & =\frac{4\left(a^{*}+a^{E U}-p\right)}{16-p^{2}\left(a^{*}+a^{E U}-2 p+2 \widetilde{p}+t\right)}, \\
\widehat{c}_{W} & =\frac{4\left(a^{*}+a^{E U}-p+2 \widetilde{p}\right)}{16-p^{2}\left(a^{*}+a^{E U}-2 p+2 \widetilde{p}+t\right)},
\end{aligned}
$$

if the environmental cost is quadratic.

Proposition 4 suggests that there can be two scenarios for the surplus and welfare effects of EU regulation. First, the parameters maybe such that regulation is always surplus and/or 
welfare enhancing. Second, EU regulation may be surplus and/or welfare increasing provided that the environmental externality of not separating waste is sufficiently strong. More precisely, the parameter $c$ has to exceed positive thresholds, $\widehat{c}_{T S}$ and $\widehat{c}_{W}$, in order to ensure that the incremental costs of effort and investment imposed by EU regulation are more than offset by the reduced cost of the externality imposed to society by unseparated waste. These results hold independently of the functional form adopted for the externality costs linked to the disposal of unseparated waste. However, the thresholds, if any, needed for EU regulation to be desirable are ceteris paribus lower if the social costs of unseparated waste grows quadratically rather than linearly. Finally, it is possible that the parameter configuration is such that surplus is always enhanced by regulation but not welfare: this is more likely when the price obtained from recycled materials, $\widetilde{p}$, is relatively high.

\section{Discussion}

This paper provides two main contributions. The first it is to formalize through a simple spatial model the economics issues related to waste collection, with a particular focus on the MSW sector in the EU. The second is to highlight the main economic effects that the EU regulation of the waste sector has on market outcomes. In particular, the model captures the fact that the SSP and PP principles, on which the current EU regulation on waste collection is based, may have an effect on the disposal prices. This price effect operates as regulation limits competition between disposers in different areas. Our main result suggests that an intuitive, pro-competitive effect may operate if regulation is abandoned: this is likely to be the case not only when areas are very similar but also when they are rather heterogeneous. Only if areas are very heterogeneous the pro-competitive effect of opening to disposers from other areas may not reduce the prices. The latter conclusion is of interest as it may allow to better understand the implications of increasing competition in the MSW sector even beyond the EU borders: for example, our findings cast doubts about the supposed unambiguous relationship between allowing waste trade and the creation of international pollution havens in developing countries (Jha et al. 1999). The intuitive pro-competitive effect that we highlighted, however, has another possible consequence: more competitive disposal markets reduce the costs of collection and that, in turn, leads to lower incentives to build selection capacity and to reduced incentives for households to engage in separated collection. Regulation, then, may encourage recycling and provide incentives for collectors and households to engage in more separated collection.

The latter effect on separated collection incentives may be one more possible rationale behind the current EU regulation. One view, popularized by Hotelling (1931) and Buchanan (1969), asserts that less competitive market structures may be desirable, in some cases, to limit negative environmental externalities. ${ }^{15}$ We highlight a similar effect within a model that captures the complicated structure of the waste collection and disposal sector. The EU policy, in this context, seems to have one more advantage, beyond its usually declared objectives (preventing the creation of pollution havens, above all): increasing the local communities' incentives to engage in recycling and separated collection.

Whereas our model has attempted to capture the main features of the MSW sector, our results were obtained under a number of simplifying assumptions. For example, as already underlined, our model focuses on a very specific channel through which the price difference

15 "It has been argued that market imperfection especially the polar case of monopoly, is the conservationists' best friend" (Hotelling 1931). 
induced by regulation matters: separated collection and recycling. To give maximal emphasis the role of this channel, other very relevant implications of price differences have been neglected in our model, above all the illegal disposal of waste. Illegal dumping is more likely to take place if regulation increases the costs of disposal and, in our model, this is the case if areas are not too heterogeneous. The surplus and welfare analysis in Sects. 4 and 5.1 is only aimed to highlight the net effect of regulation through the separated waste channel. An encompassing welfare analysis would also need to take into account the additional implications of regulation on illegal dumping: this is particularly relevant if the model was to be adopted to obtain more precise policy indications. Furthermore, one of the assumptions of our model is that the regime does not influence the regulated price of collection of both the separated, $\tilde{p}$, and the unseparated waste, $p$. The price of unseparated collection at least is likely to be related to the price of disposal. One way of capturing such a feature in our model is, for example, by assuming that the regulator applies a fixed mark-up to set the price of unseparated collection, e.g. $p_{i}=\mu_{i} a_{i}$ with $\mu_{i}>1$. Such a case is tractable and it is easy to verify that the equilibrium expressions, substituting the relations above, are unaffected up to stage 2 , i.e. up to the collectors' choices. However, as the solution of the first stage, in which prices of the disposers are chosen, is less straightforward and the expressions are rather complicated, in the paper we choose to focus on the basic case. Our results, however, turn out to be even sharper in the extended model. The reason is rather intuitive: abandoning the current regulatory regime, in fact, leads also to an adjustment in the relative price of unseparated collection. The pro-competitive effect, then, implies a lower disposal price but also, proportionally, even less separated waste. A similar effect is likely to operate also in case the total amount of waste produced by the households in a municipality, that we normalised to one in our model, is allowed to vary between regimes: relaxing the current regulation would lead to the lower prices in the unseparated section of the market and this is likely to increase the size of the overall waste produced.

Open Access This article is distributed under the terms of the Creative Commons Attribution 4.0 International License (http://creativecommons.org/licenses/by/4.0/), which permits unrestricted use, distribution, and reproduction in any medium, provided you give appropriate credit to the original author(s) and the source, provide a link to the Creative Commons license, and indicate if changes were made.

\section{A Appendix}

\section{A.1 Proof of Lemma 1}

If EU regulation is in place, the first order conditions are symmetric for the two areas and can be written as:

$$
\operatorname{FOC}_{i}^{E U}\left(a_{i}\right)=q_{i}^{E U}+a_{i} \frac{\partial q_{i}^{E U}}{\partial a_{i}}=0
$$

Importantly, the first order condition is unaffected by the variables and parameters relating to area $j$. As $\partial q_{i}^{E U} / \partial a_{i}=-p_{i}^{2} / 8<0$, then $F O C_{i}^{E U}\left(a_{i}\right)$ is decreasing in $a_{i}$ and a unique equilibrium exists. Substituting the relevant expressions, the equilibrium can also be characterized as (3.13). 


\section{A.2 Proof of Proposition 2}

The effect of abandoning the current EU regulation is considered. Given our assumption on $x_{A B}^{*}$, there are two possible cases, depending on the "fundamentals" (i.e. $p_{i}$ and $\tilde{p}_{i}$ ): (a) the equilibrium is such that $a_{A}^{E U}=a_{B}^{E U}$; (b) the equilibrium is such that $a_{A}^{E U}<a_{B}^{E U} \cdot{ }^{16} \mathrm{We}$ shall consider each in turn, corresponding to parts (a) and (b) of the Proposition.

(a) If $a_{A}^{E U}=a_{B}^{E U}$ and regulation stops being enforced, according to (3.3) we shall still have $x_{A B}^{*}=1$. Suppose disposer $A$ considers decreasing the price from $a_{A}^{E U}$; in that case the relevant profit function is (3.10), as $x_{A B}^{*}>1$ following the considered price decrease. The impact of the change in the price, evaluated at $a_{A}^{E U}=a_{B}^{E U}$, can be written as:

$$
\left.\frac{\partial \pi_{A}^{D}}{\partial a_{A}}\right|_{a_{A}=a_{A}^{E U}}=\underbrace{F O C_{A}^{E U}\left(a_{A}^{E U}\right)+q_{B}^{E U}\left(x_{A B}^{*}-1\right)+a_{A}^{E U} \frac{\partial q_{B}}{\partial a_{A}}\left(x_{A B}^{*}-1\right)}_{=0}+\underbrace{a_{A}^{E U} q_{B}^{E U} \frac{\partial x_{A B}^{*}}{\partial a_{A}}}_{-}<0,
$$

where the first three terms are zero as a consequence of $\partial \pi_{A}^{D} / \partial a_{A}$ being evaluated at the EU equilibrium. Hence, (A.2) implies that a unilateral price decrease, increases disposer A profits. A similar argument applies to disposer $B$. These imply that $a_{i}^{*}<a_{i}^{E U}$.

(b) If $a_{A}^{E U}<a_{B}^{E U}$ then abandoning regulation would imply $x_{A B}^{*}>1$ even if no price adjustment is made. From the perspective of disposer $A,(3.10)$ is still the relevant profit function and the impact of a change in price is still:

$$
\left.\frac{\partial \pi_{A}^{D}}{\partial a_{A}}\right|_{a_{A}=a_{A}^{E U}}=\underbrace{F O C_{A}^{E U}\left(a_{A}^{E U}\right)}_{=0}+\underbrace{q_{B}^{E U}\left(x_{A B}^{*}-1\right)}_{+}+\underbrace{a_{A}^{E U} \frac{\partial q_{B}}{\partial a_{A}}\left(x_{A B}^{*}-1\right)+a_{A}^{E U} q_{B}^{E U} \frac{\partial x_{A B}^{*}}{\partial a_{A}}}_{-} .
$$

In this case, however, the second term is positive as $x_{A B}^{*}>1$, whereas the last two terms are negative. The sign of (A.3) then depends on:

$$
\Phi=q_{B}^{E U}\left[x_{A B}^{*}-1+a_{A}^{E U} \frac{\partial x_{A B}^{*}}{\partial a_{A}}\right]+a_{A}^{E U} \frac{\partial q_{B}}{\partial a_{A}}\left(x_{A B}^{*}-1\right),
$$

and although the expression is hard to sign in general, after substitution it can be re-written as:

$$
\Phi=q_{B}^{E U}\left[\frac{1}{2 t}\left(a_{B}^{E U}-2 a_{A}^{E U}\right)\right]-\frac{a_{A}^{E U} p_{B}^{2}}{32 t^{2}}\left(a_{A}^{E U}-a_{B}^{E U}\right)^{2},
$$

implying that $a_{A}^{E U}>a_{B}^{E U} / 2$ is sufficient for $\Phi<0$ and, as a consequence, for $a_{A}^{*}<a_{A}^{E U}$.

From the point of view of disposer $B$ abandoning regulation would imply a decrease in the demand $\left(2-x_{A B}^{*}<1\right)$ even if no price adjustment is made. The relevant profits are then (3.11) and the impact of a change in price, evaluated at the EU regulation equilibrium, can be written as:

$$
\left.\frac{\partial \pi_{B}^{D}}{\partial a_{B}}\right|_{a_{B}=a_{B}^{E U}}=\underbrace{q_{B}^{E U}\left(2-x_{A B}^{*}\right)+a_{B}^{E U} \frac{\partial q_{B}}{\partial a_{B}}\left(2-x_{A B}^{*}\right)}_{<F O C_{B}^{E U}\left(a_{B}\right)}-\underbrace{a_{B}^{E U} q_{B}^{E U} \frac{\partial x_{A B}^{*}}{\partial a_{B}}}_{-}<0
$$

16 Notice that if the equilibrium is such that $a_{A}^{E U}>a_{B}^{E U}$ and $x_{A B}^{*}<1$, the proof follows from case (b) inverting the roles of $A$ and $B$. 
The latter inequality holds as the first two terms are lower than the equivalent terms in (A.1) as $2-x_{A B}^{*}<1$, whereas the last term is negative. As such, disposer $B$ always reduces his price if regulation is lifted: $a_{B}^{*}<a_{B}^{E U}$.

(c) If abandoning regulation leads to a decrease in disposal prices $a_{i}^{*}$, then the results on selection capacity, effort and overall amount of separated collection follow directly from the results obtained at Stage 2 and 3. In particular, as $\frac{\partial k_{i}^{*}}{\partial a_{i}^{*}}>0$ a lower disposal price implies a lower selection capacity; this, in turn, implies:

$$
\frac{\partial e_{i}^{*}}{\partial k_{i}^{*}} \frac{\partial k_{i}^{*}}{\partial a_{i}^{*}}>0 \text { and } \frac{\partial d_{i}^{*}}{\partial k_{i}^{*}} \frac{\partial k_{i}^{*}}{\partial a_{i}^{*}}>0
$$

\section{A.3 Proof of Corollary 3}

The claim follows directly from part (a) of Proposition 2. However, as the equilibrium is fully characterized, it is sufficient to compute the disposal price differential:

$$
\Delta a=a^{*}-a^{E U}=2 t-\frac{\sqrt{4\left(p^{3}-p^{2} \tilde{p}+8\right)^{2}-4 p^{2} t\left(p^{3}-p^{2} \tilde{p}+8\right)+65 p^{4} t^{2}}}{4 p^{2}} .
$$

For given $p$ and $\tilde{p}$, it can be verified that the function $\Delta a(t)$ has only one zero, $\widehat{t}=$ $\frac{2}{p^{2}}\left(p^{3}-p^{2} \tilde{p}+8\right)$. Hence, for all other values of $t \neq \widehat{t}, \Delta a(t)$ is either always positive or always negative. It is also straightforward to show that $\Delta a(t)$ is concave in $t$, as $\partial^{2} \Delta a / \partial t^{2} \leq 0$, implying that $\Delta a$ is always negative for $t \neq \widehat{t}$ and, consequently, $a^{*}<a^{E U}$. The implications of the result on selection capacity follow directly from part (c) of Proposition 2.

\section{A.4 Proof of Proposition 4}

Substituting the respective functional forms, (5.1) and (5.2) can be written as:

$$
\begin{aligned}
T S^{E U}-T S^{*} & =\frac{p^{2}}{8}\left(a^{*}-a^{E U}\right)\left(a^{E U}+a^{*}-p-2 c\right), \\
W^{E U}-W^{*} & =\frac{p^{2}}{8}\left(a^{*}-a^{E U}\right)\left(a^{E U}+a^{*}+2 \tilde{p}-p-2 c\right) .
\end{aligned}
$$

if the externality cost is linear and:

$$
\begin{aligned}
T S^{E U}-T S^{*}= & \frac{p^{2}}{32}\left(a^{*}-a^{E U}\right)\left[\left(a^{E U}+a^{*}\right)\left(4+c p^{2}\right)+c p^{2}(2+t-p)-4 p-16 c\right], \\
W^{E U}-W^{*}= & \frac{p^{2}}{32}\left(a^{*}-a^{E U}\right) \\
& \times\left[\left(a^{E U}+a^{*}\right)\left(4+c p^{2}\right)+c p^{2}(2+t-p)+8 \widetilde{p}-4 p-16 c\right] .
\end{aligned}
$$

if the externality cost is quadratic. In both cases, solving $T S^{E U}-T S^{*}=0$ and $W^{E U}-W^{*}=$ 0 allows to obtain, respectively, the thresholds $\widehat{c}_{T S}$ and $\widehat{c}_{W}$. As all functions are increasing in $c$, if a threshold is negative or zero, the differential is positive for all admissible values of $c$; if a threshold is positive, the differentials are positive if $c$ exceeds it. 


\section{References}

Almer C, Goeschl T (2010) Environmental crime and punishment: empirical evidence from the German penal code. Land Econ 86(4):707-726

Buchanan JM (1969) External diseconomies, corrective taxes and market structure. Am Econ Rev 59:174-177

Caplan AJ, Grijalva T, Jackson-Smith D (2006) Using choice question formats to determine compensable values: the case of landfill-siting process. Ecol Econ 60:834-846

Choe C, Fraser I (1998) The economics of household waste management: a review. Aust J Agri Resour Econ 42(3):269-302

Choe C, Fraser I (1999) An economic analysis of household waste management. J Environ Econ Manag 38(2):234-246

Crocker KJ, Masten SE (1996) Regulation and administered contracts revisited: lessons from transaction-cost economics for public utility regulation. J Regul Econ 9(1):5-39

Czajkowski M, Hanley N, Nyborg K (2016) Social norms, morals and self-interest as determinants of proenvironment behaviours: the case of household recycling. Environ Resour Econ 66(4):647-670

D'Alisa G, Walter M, Burgalassi D, Healy H (2010) Conflicts in Campania: waste emergency or crisis of democracy. Ecol Econ 70(2):239-249

D'Amato A, Zoli M (2012) Illegal waste disposal in the time of the Mafia: a tale of enforcement and social well-being. J Environ Plan Manag 55(5):637-655

Davies B, Doble M (2004) The development and implementation of a landfill tax in the UK. In: Addressing the economics of waste. OECD Publishing, Paris, pp 63-80

Demsetz H (1968) Why regulate utilities? J Law Econ 11(1):55-65

Di Corato L, Montinari N (2014) Flexible waste management under uncertainty. Eur J Oper Res 234(1):174185

Fullerton D, Kinnaman TC (1995) Garbage, recycling and illicit burning or dumping. J Environ Econ Manag 29:78-91

Hong S, Adams RM, Love HA (1993) An economic analysis of household recycling of solid wastes: the case of Portland, Oregon. J Environ Econ Manag 25(2):136-146

Hotelling H (1929) Stability in competition. Econ J 39(153):41-57

Hotelling H (1931) The economics of exhaustible resources. J Polit Econ 39(2):137-175

Jenkins RR, Maguire KB, Morgan CL (2004) Host community compensation and municipal solid waste landfills. Land Econ 80(4):513-528

Jha V, Markandya A, Vossenaar R (1999) Reconciling trade and the environment: lessons from case studies in developing countries. Edward Elgar, Cheltenham

Laffont JJ, Tirole J (1994) A theory of incentives in procurement and regulation. MIT Press, Cambridge

Lederer PJ, Hurter AP (1986) Competition of firms: discriminatory pricing and location. Econometrica 54(3):623-640

Massarutto A (2007) Municipal waste management as a local utility: options for competition in an environmentally-regulated industry. Utilit Policy 15(1):9-19

Porter ME, van der Linde C (1995) Toward a new conception of the environment-competitiveness relationship. J Econ Perspect 9(4):97-118

Reichenbach J (2008) Status and prospects of pay-as-you-throw in Europe-a review of pilot research and implementation studies. Waste Manag 28(12):2809-2814

Shinkuma T (2003) On the second-best policy of household waste recycling. Environ Resour Econ 24(1):77-95

Silvestri F (2014) Competition and environmental externalities in the European market of municipal waste. SEEDS Working Paper, 11

Silvestri F, Ghinoi S (2015) Municipal waste selection and disposal: evidences from Lombardy. FEEM Working Paper, 14

Vogel J (2011) Spatial price discrimination with heterogeneous firms. J Ind Econ 59(4):661-676

Williamson O (1976) Franchise bidding for natural monopolies-in general and with respect to CATV. Bell J Econ 7(1):73-104

Yang HL, Innes R (2007) Economic incentives and residential waste management in Taiwan: an empirical investigation. Environ Resour Econ 37:489-519 